\title{
ГРАММАТИКА КОНСТРУКЦИИ КАК ЭЛЕМЕНТ ВЗАИМОДЕЙСТВИЯ ФРАЗЕОЛОГИЧЕСКИХ ЕДИНИЦ
}

\section{CONSTRUCTION GRAMMAR \\ AS AN ELEMENT OF INTERACTION OF PHRASEOLOGICAL UNITS}

\section{Ramírez Rodríguez Pablo}

Summary: As its already known, phraseological objects are complex objects that present a number of difficulties, first, for their lexicographic description, and secondly, when trying to embed them in general ideas about the structure of the language. For this reason, the main task in this paper is to highlight the importance of the pragmatic element of interaction of phraseological units used in the spoken Spanish language.

Keywords: construction grammar, phraseological units, pragmatics, interaction, colloquial speech.
Рамирес Родригес Пабло

Аспирант, РУДН, Москва

ramires-rodriges-p@rudn.ru

Аннотация: Как известно, фразеологические единицы являются сложными объектами, представляющими ряд трудностей, во-первых, для их лексикографического описания, а во-вторых, при попытке встроить их в общие представления 06 устройстве языка. По этой причине, задача в данной работе заключается в том, чтобы выделить важность прагматического элемента взаимодействия фразеологических единиц употребляющихся в устной речи испанского языка.

Ключевые слова: грамматика конструкции, фразеологические единицы, прагматика, взаимодействие, разговорная речь.

дискурса, таких как называемых разговорные формулы.

Среди этих фразеологических высказываний находится подкласс, состоящий из формул, хорошо известных носителям любого языка, поскольку к ним часто прибегают в повседневной жизни. Эти фразеологические единицы получили в течение истории лингвистических исследований различные конфессии, такие как: ритуальные высказывания (Lyons: 1968), формулы вежливости (Ferguson: 1976), рутинные формулы (Coulmas: 1979) и прагматические формулы фиксачии (Zuluaga: 1980).

Таким образом, формулы - это готовые заявления, к которым обращаются ораторы, когда ситуация оправдывает их. Правда, что не все из нас участвуют в одинаковых социально-культурных мероприятиях. Все зависит от типа говорящего, личной или профессиональной ситуации и других факторов. Тем не менее, хорошо известно, что все практикуют лексику разговорной речи, так как это единственная словесная деятельность, которую все члены сообщества разделяют естественным образом и которая характеризуется своей спонтанностью. В свою очередь, она имеет большой набор выражений, которые люди используют обычным способом для выполнения определенных ритуальных речевых актов, таких как благодарность, поздравление, удивление, отказ или упрек.

Дискурсивная формула в русском языке «да ну!» употребляется при выражении удивления на какую-то новость (1), а также в контексте отказа на какое-то пред- 
ложение (2), между прочем. Однако, в испанском языке употребляются разные формулы для каждого контекста, так как оба контекста выражают противоположные эмоции. В первом примере испанская формула venga уа употребляется при удивлении или сомнения, в то время как si hombre выражает полное отрицание или несогласие.

1. Я дописал сегодня диссертацию! - да ну! Хочу ее чиmamb. (¡He terminando de escribir la tesis! - ivenga ya! [букв. Давай уже] Quiero leerla)

2. Надо написать сегодня отчёт по практике - да ну! Завтра мы его напишем, давай отдохнём сейчас. (Нау que escribir el informe de las prácticas hoy- ¡Si hombre! [букв. Да мужчина] Mañana lo hacemos, vamos mejor a descansar ahora)

Есть две причины, побудившие интерес к этому типу языковых единиц. С одной стороны, автор работы не является носителем русского языка, что позволил ему оценить эти выражения, к которым без затруднений прибегают носители языка в практике разговорной речи, и которые, несомненно, представляют серьезное препятствие для не носителей языка. С другой стороны, его профессиональный опыт преподавания испанского языка как иностранного для студентов-переводчиков привел к наблюдению, что существует внутренняя трудность в расшифровке и переформулировании единиц этого типа, для которых должны быть предложены функциональные и прагматические эквиваленты, а не только лингвистический.

Несмотря на трудности, связанные с переводом, именно из-за прагматически-функциональных аспектов языка и культуры происхождения, а также целевого языка и культуры этот класс высказываний не описан полностью и однородно в грамматиках испанского или русского языков, в учебниках по иностранному языку или в двуязычных словарях. Именно поэтому необходимо провести сравнительный анализ двух изучаемых языков.

Таким образом, общая цель этой работы состоит в том, чтобы ответить на практическую задачу, предоставить переводчику, лексикографу и учителю на языках необходимые инструменты для решения проблем понимания, производства и переформулирования, которые ставят формулы разговора.

Многие из нынешних авторов за последние двадцать лет показывали (Vigara: 1992, Briz: 1996 или Montolío: 1996), что грамматический анализ разговорного устного дискурса передает другое изображение традиционного исследования, посвященного формальному синтаксису. Априори, с более методологической точки зрения, можно выделить два аспекта, которые затрудняют обработку грамматических структур, используемых в разговорной среде, они приближаются к компонентам, предлагающим автономный синтаксический компонент, то есть компонент нечувствительный к просодии, к семантическо-прагматике и к речи. Во-первых, грамматичность спонтанной устной речи зависит не только от морфосинтактических аспектов, но и от просодической и дискурсивной информации (Narbona: 2000).

Описание, начинающееся только с морфосинтактической основы недостаточно, так как оно может привести к неграмматической последовательности. По этой причине морфосинтактическая информация должна быть заполнена просодической информацией.

Также большинство структур, приведенных в спонтанном устном языке, находятся в рамках выражения прагматических функций. То есть нужно подумать о том, что такое лингвистическая форма, выданная говорящим, и каков вклад в интерпретацию высказывания. Синтаксическая конструкция всегда включает в себя дискурсивную информацию (Montolío: 1996).

Согласно моделям, описанных в книгах «Principles and parameters» 'Принципы и параметры' (Chomsky, Lasnik: 1995) и «Programa minimalista» 'Минимистическая программа' (Chomsky: 1999), подробный анализ грамматических конструкций должен раскрывать более общие принципы функционирования языка, так что взаимодействие интересов формальных аспектов, по-видимому, расходятся. Это объясняет, почему лингвисты, посвященные описанию грамматических структур во взаимодействии, предпочли обратить свое внимание на другие теории в области прагматики, анализ дискурса или анализ речи.

Самые плодотворные исследования из лингвистических теорий даны в областях: дискурсивных маркеров, которые создают впечатление о существовании двух типов грамматик: обычная грамматика, составлена из общих конструкций языка, а также периферическая грамматика, которая составлена из конструкций с точки зрения прагматики и дискурса.

Концепция для многих лингвистов с когнитивнофункциональной точки зрения - это знание языка, то есть знание об использовании языка (Cuenca: 2003) В этом смысле грамматика конструкций (Goldberg: 2006; Kay, Fillmore: 1999) может соответствовать такому описанию. Чтобы составить краткое представление о термине грамматика конструкций, необходимо дать его определение. Грамматика конструкций - это набор предложений, которые имеют одну и ту же цель: требование к грамматической конструкции как основная единица лингвистического анализа. 
Такие грамматические конструкции определяются как стабильные формальные и интерпретирующие элементы с разной степенью структурной сложности и схематичности. Согласно этому подходу, лингвистическое знание говорящего описывается как система сложных конструкций, в которых вмешиваются лексика, синтаксис, фразеология и даже дискурсивные шаблоны. Поэтому проводится различие между центральными и периферийными структурами в зависимости от их важности в построении грамматики данного языка.

Холистическая концепция конструкций вместе с обязательством охватить все структуры языка, независимо от степени общности или идиоматичности, делает грамматику конструкций подходящей моделью для описания структур, находящихся во взаимодействии (Linell: 2009). Анализ грамматических структур в ситуации устного взаимодействия показывает исследовательские, лексические и синтагматические категории, которые едва ли были изучены традициями. Среди этих структур: маркеры дискурса, используемые в качестве инструментов, с помощью которых говорящие выполняют акт коммуникации через функции соединения, информацию о дискурсе, выражение режима или контактный контроль между собеседниками.

В определенном смысле «Грамматика Конструкций» предполагает возвращение к традиционным позициям (Lakoff: 1987; Garachana, Hilferty: 2005), поскольку он преследует цель интегрировать анализ формы и смысла грамматических структур. Однако, для «Грамматики Конструкций» грамматические конструкции не являются чисто формальными, а являются элементами, которые объединяют формальные (фонологические, морфологические и синтаксические) и функциональные (семантические и прагматические) особенности.

Как известно, лингвистические знания говорящего состоят из организованного репертуара грамматических конструкций, будь то простые, общие или сложные. Однако, конструктивистские подходы пытаются, в отличие от традиционных грамматик, выделить механизмы, позволяющие обобщить конкретные лингвистические выражения (Lakoff: 1987).

Что касается происхождения «Грамматики Конструкций», учитывая его внешний вид, трудно прояснить и наметить рождение этой теории. Тем не менее, мы можем ответить за гестацию этой теории, основанной в Калифорнийском университете в Беркли, США, в восьмидесятые годы Чарльза Дж. Филлмора и Джорджа Лакоффа.

Оба автора выступали за создание генеративного семантика (Generative Semantics), течение, которое возникло из Генеративной Грамматики (Generative Grammar) в шестидесятых годах, чья максима заключалась в том, чтобы разместить значение в центре исследований, а также интегрировать в семантический компонент теорий вклады о прагматике, речевых актов или логике разговора.

Первые работы предлагали явно конструктивный подход к грамматике (Fillmore: 1989; Fillmore, Kay: 1988; Lakoff: 1987). В наши дни используются несколько подходов, в частности, два конструкционистских аспекта, предложенных C.J. Fillmore с целью формализации формальных и функциональных особенностей общих и идиоматических конструкций; а также предложение G. Lakoff, в котором основное внимание уделяется роли категоризации в грамматических знаниях.

Чтобы найти Грамматику Конструкций в текущей лингвистической панораме, необходимо проследить за совпадающими и расходящимися точками с формалистическими подходами и за когнитивными и функциональными подходами (Croft: 2001). Современная лингвистика предполагает, что языки не могут быть описаны просто как совокупность явлений, поскольку они должны рассматриваться как внутренние системы, используемые говорящими. Кроме того, говорящие языка могут использовать воображение для формирования новых утверждений из комбинации существующих элементов, поэтому язык является сложным механизмом, который позволяет комбинировать элементы для формирования высказываний.

В настоящее время лингвистическое мышление имеет тенденцию устанавливать строгое разделение между знаниями, которыми владеют говорящие, и тем, как эти говорящие используют эти знания. Это разделение восходит к лингвистическому структурализму через известное противостояние langue у parole 'компетентностью и исполнением' и консолидируется в генеративизме через противостояние между конкуренцией и исполнением.

Конструкционистский подход подстраивается под сомнение удобство построения модели грамматики независимо от использования этой грамматики говорящими. С одной стороны, некоторые конструкционистские подходы утверждают необходимость включения прямого опыта говорящих в использовании этих форм в когнитивное представление лингвистических форм. С другой стороны, конструкционистские подходы ставят под вопрос разделение компетенции и исполнении на то, что некоторые грамматические конструкции традиционно используются с точной прагматической функцией (Fillmore: 1989). С этой точки зрения анализ некоторых конструкций требует восстановления понятий из исследования взаимодействия, в основном из прагматики, и из дискурсивного и речевого анализов (Langacker: 1987).

Одним из наиболее привлекательных аспектов грамматики конструкций для анализа грамматических струк- 
тур в ситуации взаимодействия является включение прагматического измерения, необходимого из-за существования прагматической информации, закодированой в синтаксических структурах.

Формальные подходы к прагматике установили четкое разделение между грамматикой и прагматикой (Fillmore: 2006). С одной стороны, грамматика связана с объяснением значений независимо от контекста, использования или конечной цели. С другой стороны, прагматика это прежде всего механизмы, которые объясняют намерения, с помощью которых говорящие используют языковые выражения в конкретных контекстах.

С этой точки зрения, прагматика должна учитывать механизмы толкования, основанные на locall and special cultural conventions 'локальный и специальных культурных конвенциях', например: испанская конвенция как вежливо отклонять приглашение, и логические механизмы, основанные на common-sense reasoning 'здравом смысле'. Это разговорная импликация представлена в примере (3).

3. A: ¿Tienes fuego? 'У тебя есть зажигалка?' (букв. у тебя есть огонь)

\section{B: No fumo. 'Я не курю.'}

Чтобы охарактеризовать прагматическую ценность грамматических конструкций, необходимо ответить на два вопроса: какие аспекты интерпретации традиционно связаны с грамматической конструкцией и какие теоретические инструменты необходимы для исчерпывающего описания типа вклада, который делает построение для интерпретации высказывания (типы контекстов и лингвистических функций).

Что касается типов прагматической информации, которые могут быть выражены через грамматические конструкции, то нет определенного предложения. По этой причине «Грамматика Конструкций» должна обратиться к исследованиям в прагматике, анализе дискурса и анализе разговоров, чтобы включить категории, которые позволяют точно описать контексты использования конструкций.

Что касается отношения между грамматическими построениями и единицами разговора, анализируется взаимосвязь между анафорическими выражениями и диалоговыми единицами и предполагается, что выбор определяется последовательностью разговорной единицы. Аналогично, анализируется использование наречных предложений при взаимодействии и определяется, что позиция (начальная или конечная) этих структур коррелирует с проекцией и расширением диалоговых поворотов (Ford: 1993). Также существует способ ограни- чения ценности многозначных маркеров дискурса, объединяя понятие грамматической конструкции и единицы разговора. Что касается взаимосвязи между грамматическими конструкциями и интерактивными действиями, некоторые частые интерактивные рутины соотносятся с определенными лингвистическими формами.

Грамматическая конструкция определяется как единое целое, поскольку это рутина, хранящаяся в памяти говорящего; это общепринято, учитывая, что его значение дается консенсусом определенного сообщества говорящих и является символическим, поскольку оно состоит из систематической ассоциации формального представления с семантическим представлением. В этом смысле Грамматика Конструкций представляет собой попытку описать лингвистическое знание говорящих в их совокупности из концепции грамматической конструкции.

В этой работе подчеркивается, что особые отношения, которые устанавливаются между интонацией, морфосинтаксом и значением во многих интерактивных построениях, в полной мере согласуются с предпосылками конструктивного подхода к грамматике. Таким образом, адекватное описание многих грамматических структур взаимодействия требует, помимо инструментов грамматики, учитывать информацию, связанную с различными лингвистическими дисциплинами (фонетика и фонология, семантика и прагматика). Эта ситуация, вопреки ожиданиям модульного подхода к языку, соответствует предпосылкам «Грамматики Конструкций». Цель данной работы - показать важность адекватности конструктивного подхода к языку для достижения глобального понимания грамматических структур взаимодействия, таких как дискурсивные формулы разговорной речи.

\section{Зак^ючение}

Разговор - это одно из важнейших коммуникативных событий, которые развивает человек. По словам Т. A. Van Dijk, «разговор имеет свою собственную грамматику с синтаксическими правилами, характерными для устной модальности языка» (Van Dijk: 1983). В этом случае можно выделить различные типы синтаксических и конструкционных стратегий, такие как сцепленный синтаксис, избыточность, объяснительный обход, открытое объединение, прагматический порядок и соединение через прагматические и интонационные соединители.

Кроме того, чтобы разговор состоялся не только по очереди, но и для каждого речевого акта, каждое выступление оратора должно быть связано с высказываниями и предыдущими выступлениями его и других людей, предвосхищая их толкования и ответы. В этом смысле разговор идентифицирует ряд элементов, которые, помимо придания сплоченности и, следовательно, согласованности разговору, являются признаками аргу- 
ментативной активности (аргументативные маркеры), формулировочной (метадискурсивные маркеры) и индикаторами отношение говорящего в процессе высказывания (модальные маркеры).

Изучение фразеологических единиц способствует развитию коммуникативной компетенции, которая является основной целью процесса преподавания и изучения второго языка, в данной работе рассмотрен испанский язык. Мы считаем, что учащийся осуществляет эффективное взаимодействие на испанском языке, когда его участие в коммуникативном обмене является успешным, то есть когда он использует необходимый лингвистический контент, адаптируя свою продукцию к коммуникативной ситуации и принимая во внимание контекст. В этом смысле, для того чтобы общение было эффективным, важно, чтобы выбор лексических единиц, в данном случае обычных формул, которые выполняет учащийся, соответствовал лингвистическим функциям, которые происходят в этом взаимодействии с социокультурной, прагматичной и дискурсивной точки зрения.

С другой стороны, необходимо помнить, что этот успех во взаимодействии, в свою очередь, усилит эффективный компонент обучения, который непосредственно повлияет на улучшение процесса обучения и представление ученика о себе как о говорящем.

Исходя из идеи, что говорить на другом языке означает действовать в другом культурном контексте следует, что язык и культура составляют единую неделимую сущность. Кроме того, мы должны осознавать, что в классе ELE всегда присутствуют две реальности: язык и культура учащегося, которые являются отправной точкой для анализа и интерпретации всего, что происходит в классе, а язык и культура цель, которая является объектом изучения и интереса, и которая постоянно ставит перед студентами языковые и культурные проблемы.

Мы должны учитывать как студенческую культуру, убеждения, языковые особенности, социокультурные привычки и обычаи, так и те, которые типичны для обществ, в которых говорят на испанском языке, и содействовать работе, в которой обе культуры присутствуют в глобальном развитии студента как говорящего на испанском языке.

\section{ЛИТЕРАТУРА}

1. Briz 1996 - Gómez, A.B., Briz, A. El español coloquial: situación y uso (Vol. 38). // Arco Libros, 1996.

2. Chomsky 1999 — Chomsky, N. El programa minimalista (Vol. 4). Alianza Editorial. 1999.

3. Chomsky, Lasnik 1995 — Chomsky, N., \& Lasnik, H. Principles and parameters. Chomsky (1995), 13-127. 1995.

4. Coulmas 1979 — Coulmas, F. On the sociolinguistic relevance of routine formulae. Journal of pragmatics, 3(3-4), 239-266. 1979.

5. Croft 2001 — Croft, W. Radical construction grammar: Syntactic theory in typological perspective. Oxford University Press on Demand. 2001.

6. Cuenca 2003 - Cuenca, M.J. Mecanismos lingüísticos y discursivos de la argumentación. Comunicación, lenguaje y educación, 7(2), 23-40. 2003.

7. Ferguson 1976 - Ferguson, C.A. The structure and use of politeness formulas. Language in society, 5(2), 137-151. 1976.

8. Fillmore 1988 - Fillmore, C.J. The mechanisms of "construction grammar". In Annual Meeting of the Berkeley Linguistics Society(Vol. 14, pp. 35-55). 1988.

9. Fillmore 1989 - Fillmore, C.J. Grammatical construction theory and the familiar dichotomies. In North-Holland Linguistic Series: Linguistic Variations (Vol. 54, pp. 17-38). Elsevier. 1989.

10. Fillmore 2006 — Fillmore, C.J. Frame semantics. Cognitive linguistics: Basic readings, 34, 373-400. 2006.

11. Ford 1993 - Ford, C.E. Grammar in interaction: Adverbial clauses in American English conversations (Vol. 9). Cambridge University Press. 1993.

12. Garachana, Hilferty 2005 - Hilferty, J., \& Garachana, M. On the reality of constructions: The Spanish reduplicative-topic construction. Annual Review of Cognitive Linguistics, 3(1), 201-215. 2005.

13. Goldberg 2006 - Goldberg, A.E. Constructions at work: The nature of generalization in language. Oxford University Press on Demand. 2006.

14. Kay, Fillmore 1999 — Kay, P., \& Fillmore, C.J. Construction grammar and linguistic generalizations: The whats X doing Y? construction. Language, 75, 1-33. 1999.

15. Lakoff 1977 — Lakoff, R. What you can do with words: Politeness, pragmatics and performatives. In Proceedings of the Texas conference on performatives, presuppositions and implicatures(pp. 79-106). 1977.

16. Langacker 1987 — Langacker, R.W. Foundations of cognitive grammar: Theoretical prerequisites (Vol. 1). Stanford university press. 1987.

17. Linell 2009 — Linell, P. Rethinking language, mind, and world dialogically. IAP. 2009.

18. Lyons 1968 - Lyons, J. Introduction to theoretical linguistics. Cambridge university press. 1968.

19. Montolío 1996 - Montolío Durán, E. Gramática e interacción. A. Briz \& al., Pragmática y grámatica. 1996.

20. Narbona 2000 - Narbona, A. Sintaxis coloquial. In Introducción a la lingüística española (pp. 463-478). 2000.

21. Van Dijk 1983 — Van Dijk, T.A. La ciencia del texto. Barcelona: Paidós. 1983.

22. Vigara 1992 - Vigara Tauste, A.M. (Morfosintaxis del español coloquial. // Madrid: Gredos, 1992.

23. Zuluaga 1980 — Zuluaga, A. Introducción al estudio de las expresiones fijas (Vol. 10). Peter Lang Publishing, Incorporated. 1980.

(c) Рамирес Родригес Пабло (ramires-rodriges-p@rudn.ru).

Журнал «Современная наука: актуальные проблемы теории и практики» 